\title{
Evaluation of a New Temporary Immersion Bioreactor System for Micropropagation of Cultivars of Eucalyptus, Birch and Fir
}

\author{
Edward Businge ${ }^{1}$, Adelina Trifonova ${ }^{2}$, Carolin Schneider ${ }^{3}$, Philipp Rödel ${ }^{3}$ \\ and Ulrika Egertsdotter 1,4,* \\ 1 Department of Forest Genetics and Plant Physiology, Umeå Plant Science Center (UPSC), \\ Swedish University of Agricultural Sciences (SLU), 90183 Umeå, Sweden; busingeed2003@gmail.com \\ 2 SweTree Technologies AB, P.O Box 4095, 90403 Umeå, Sweden; Adelina.Trifonova@swetree.com \\ 3 Institut für Pflanzenkultur e.K., Solkau 2, 29465 Schnega, Germany; schneider@pflanzenkultur.de (C.S.); \\ roedel@pflanzenkultur.de (P.R.) \\ 4 G.W. Woodruff School of Mechanical Engineering, Georgia Institute of Technology, 500 Tenth Street NW, \\ Atlanta, GA 30332-0620, USA \\ * Correspondence: Ulrika.Egertsdotter@slu.se; Tel.: +46-(0)70-4226-710 or +1-404-663-6950 \\ Academic Editor: Jason A. Holliday \\ Received: 12 May 2017; Accepted: 31 May 2017; Published: 3 June 2017
}

\begin{abstract}
The use of liquid instead of solid culture medium for the micropropagation of plants offers advantages such as better access to medium components and scalability through possible automation of the processes. The objective of this work was to compare a new temporary immersion bioreactor (TIB) to solid medium culture for the micropropagation of a selection of tree species micropropagated for commercial use: Nordmann fir (Abies nordmanniana (Steven) Spach), Eucalyptus (E. grandis $x$ E. urophylla), Downy birch (Betula pubescens Ehrh), and Curly birch (Betula pendula var. carelica). Cultivation of explants in the TIB resulted in a significant increase of multiplication rate and fresh weight of Eucalyptus and B. pendula, but not Betula pubescens. In addition, the fresh weight of embryogenic tissue and the maturation frequency of somatic embryos increased significantly when an embryogenic cell line of A. nordmanniana was cultivated in the TIB compared to solid culture medium. These results demonstrate the potential for scaling up and automating micropropagation by shoot multiplication and somatic embryogenesis in commercial tree species using a temporary immersion bioreactor.
\end{abstract}

Keywords: forest trees; temporary immersion; somatic embryogenesis; micropropagation

\section{Introduction}

In vitro culture systems based on liquid culture medium are considered to be more effective than solid culture medium systems due to better accessibility of medium components for the plant tissue, ease of handling, and possibility to scale and automate. However, as direct liquid immersion of plant material causes issues with vitrification and sometimes prohibits the growth and development of the cultured material, temporary immersion bioreactors have emerged as a workable alternative capturing the benefits of liquid medium and solid support. In comparison with both solid and liquid culture systems, temporary immersion bioreactor systems have been shown to offer technological and quantitative benefits such as higher proliferation rate and reduction of production cost [1]. For the micropropagation of both hardwood species and conifers, efficient multiplication at the in vitro stage is essential to obtain sufficient numbers of plants for production purposes. In this study, a novel model of a temporary immersion bioreactor (TIB) with an improved design for handling and different options for the optimization of growth and development was tested for basic growth and development 
through micropropagation of selected tree species of commercial value. The bioreactor has previously been demonstrated to allow for high yields, low levels of vitrification, and low contamination rates in somatic embryo cultures of Norway spruce (Picea abies (L.) Karst), attributed to the flexible culture system with respect to culture support in the bottom of the bioreactor, high rates of air exchange, and a design separating culture space from culture medium [2].

In hardwood species, micropropagation is mostly by shoot multiplication. The genus Eucalyptus comprises more than 600 tree species, most of which are native to Australia but which are grown worldwide for valuable products such as timber and essential oils [3]. Most genotypes can be readily propagated by cuttings, but some high-value genotypes are more difficult to propagate due to poor rooting caused by the low number of adventitious roots per cutting, which affects root system symmetry and tree stability [4]. In addition to offering an alternative method to clonal propagation by cuttings, in vitro cultured plant material is required as the target for biotechnological modifications. Birches (Betula spp.) are deciduous trees which are naturally distributed throughout Eurasia and are regularly used for afforestation of cleared and fire-affected forest areas $[5,6]$.

Somatic embryogenesis (SE) is an efficient method for the clonal propagation of conifers. SE plant propagation is applied in key conifer species utilized for commercial forestry production [7] as well as the production of Christmas trees (e.g., A. nordmanniana) [8-10]. The aim of this study was to evaluate a new TIB previously developed and successfully applied for Norway spruce somatic embryogenesis [2] for applicability in shoot multiplication of selected hardwood species A. nordmanniana, E. grandis $x$ E. urophylla, B. pubescens Ehrh, and B. pendula var. carelica with respect to fresh weight gain and multiplication rate; and multiplication of somatic embryos of another commercially viable conifer $A$. nordmanniana.

\section{Results}

\subsection{Effect of the TIB on Appearance, Multiplication Rate, and Fresh Weight of Explants}

In order to evaluate the applicability of the TIB for shoot multiplication, we compared it to micropropagation using solid medium culture. In terms of appearance, we observed wrinkling of leaves in the plants of $B$. pendula after 42 days of propagation in the TIB (Figure 1G,J), whereas the plants derived from solid culture medium appeared smooth and unwrinkled (Figure 1A,D). By contrast, the overall appearance of the plants of B. pubescens (Figure $1 \mathrm{~B}, \mathrm{E}, \mathrm{H}, \mathrm{K}$ ) and shoot cultures of Eucalyptus (Figure 1C,F,I,L) was normal regardless of the method of propagation. Furthermore, in contrast to the TIB, we observed actively growing roots in the plants of B. pubescens after 42 days of propagation on solid culture medium (Figure 1B,E). With regard to multiplication rate, in vitro propagation using the TIB resulted in significant increase of multiplication rate for two of the cultivars used in this study. Particularly, explants of B. pendula and Eucalyptus exhibited a 1.75- and 5.75-fold increase in multiplication rate when cultivated in the TIB compared to solid culture medium, respectively. By contrast, a 1.5-fold increase in multiplication rate was observed when the explants of B. pubescens were cultivated on solid culture medium (Figure 2a). The changes in fresh weight displayed similar patterns to those observed for multiplication rate. Generally, we observed significant increases in fresh weight when Eucalyptus and B. pendula were cultivated in the TIB. By contrast, cultivation of B. pubescens using solid culture medium led to a significant increase in fresh weight (Figure 2b). 

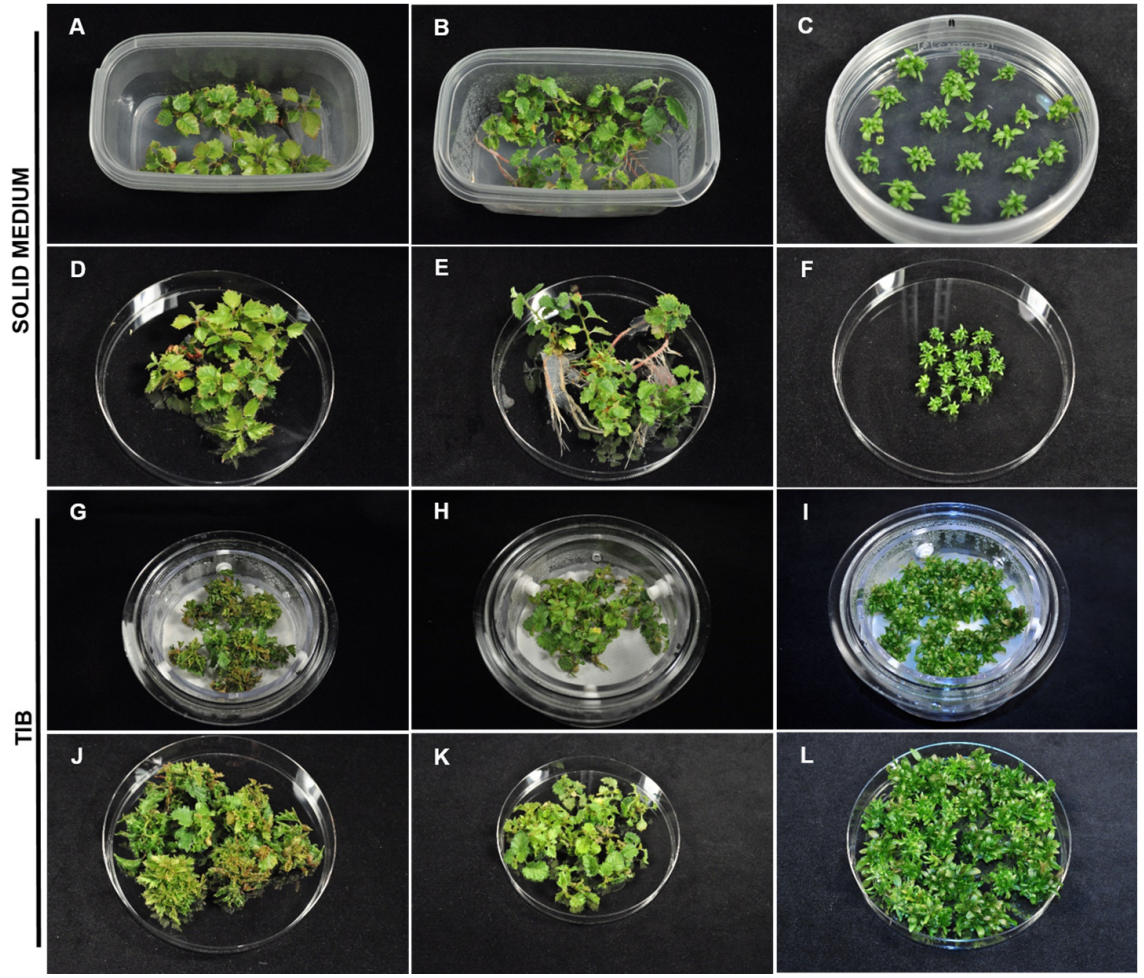

Figure 1. Plants and shoot cultures micropropagated on solid medium (top rows) and in the temporary immersion bioreactor (TIB) (bottom rows). (A,D,G,J) Plants of B. pendula after 6 weeks of culture on McCown's Woody plant medium (WPM). (B,E,H,K) Plants of B. pubescens after 6 weeks of culture on WPM medium. (C,F,I,L) Shoot cultures of Eucalyptus after 2 weeks on Murashige and Skoog (MS) medium. (D-F,J-L) represent photographs of the plants and shoot cultures outside of the propagation vessels.
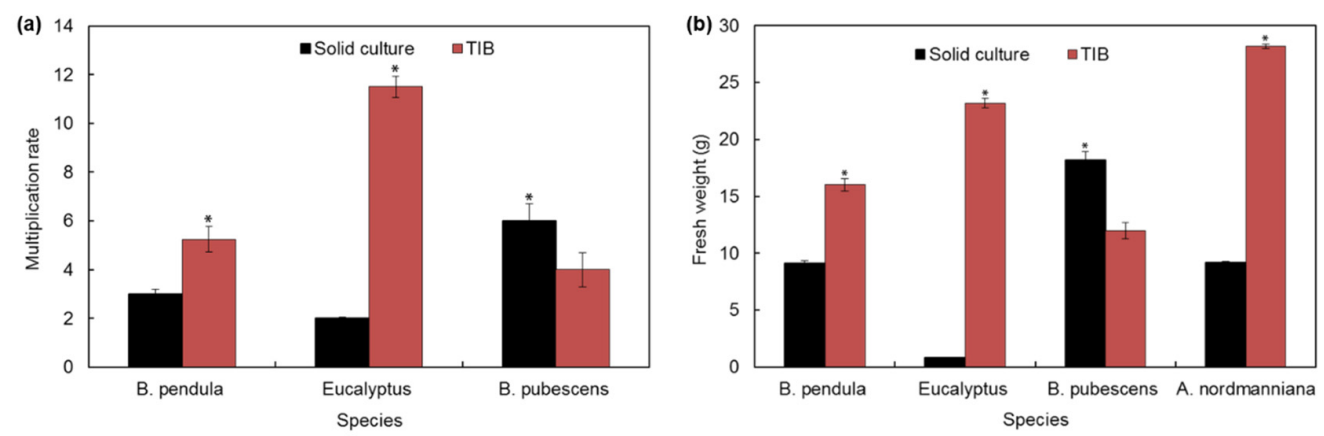

Figure 2. Effect of the TIB and solid culture medium on multiplication rate (a) and fresh weight (b). The data represents mean values \pm SEM. Each treatment comprised of three culture vessels.

\subsection{Acclimatization of in Vitro-Cultured Betula pendula and pubescens to Ex Vitro Conditions}

Acclimatization to ex vitro conditions was conducted by subjecting 12 TIB-propagated plants of B. pendula and B. pubescens to a modified version of the root induction treatment proposed by Kroin and co-workers [11]. The modified method has the potential to aid rapid clonal propagation of Betula spp. by utilizing a 12-h temporary immersion approach instead of the standard method of transferring the plants onto solid rooting medium for 4-6 weeks. After 51 days in the growth chamber, we observed that the TIB and solid medium-propagated plants of B. pendula and B. pubescens acclimated well to ex vitro conditions. They developed into healthy and morphologically normal plants with no differences in their appearance (Figure 3A-C). 

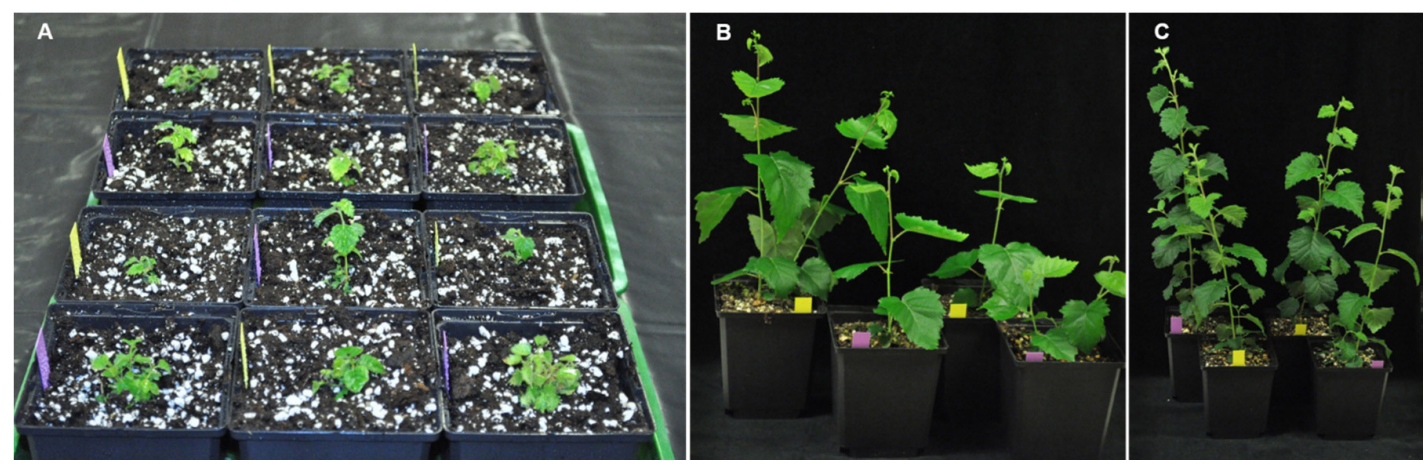

Figure 3. (A) Plants of B. pendula and B. pubescens at the start of the acclimatization period. (B,C) B. pendula and B. pubescens after 51 days of acclimatization, respectively. The purple and yellow tags indicate plants propagated on solid medium and in the TIB, respectively.

\subsection{Effect of the TIB on Fresh Weight of Embryogenic Tissue and Maturation Frequency of Somatic Embryos}

A significantly $(p=0.003)$ higher number of mature somatic embryos were obtained when A. nordmanniana cell line N.12.9/7.203 was grown in the TIB ( 185 embryos per TIB) compared to solid culture medium ( 52 embryos per petri dish) (Figure $4 \mathrm{~A}, \mathrm{C})$. The mature embryos appeared normal with split cotyledons regardless of the propagation system (Figure 4B,D). Furthermore, cell line N.12.9/7.203 produced almost three times more embryogenic tissue when cultivated in the TIB compared to solid culture medium (Figure 4C).
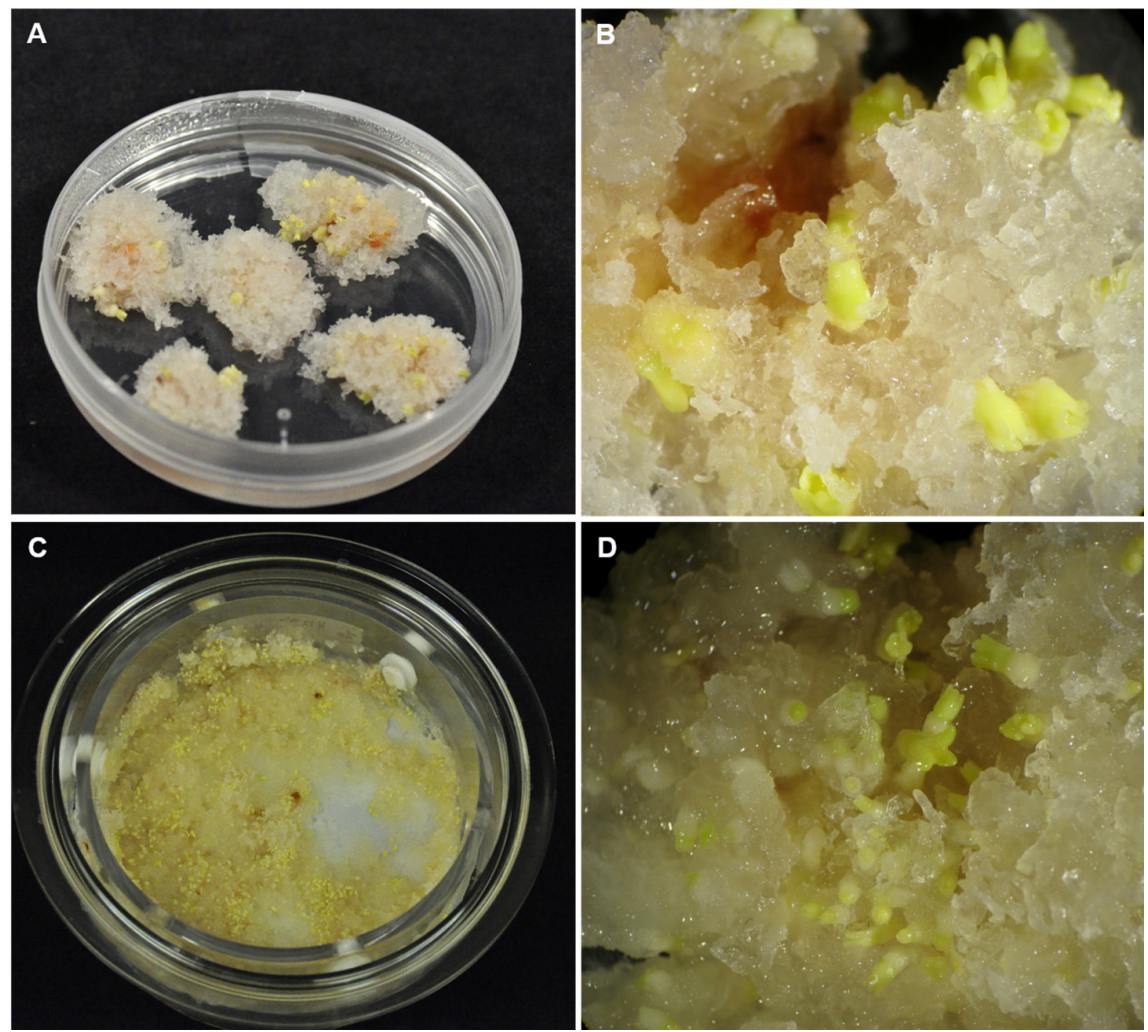

Figure 4. Somatic embryogenesis of A. nordmanniana on solid culture medium (top row) and in the TIB (bottom row). (A,D) Maturation of A. nordmanniana somatic embryos using solid and liquid Brown and Lawrence (BLG) medium supplemented with $45 \mathrm{~g} \cdot \mathrm{L}^{-1}$ maltose, $50 \mathrm{~g} \cdot \mathrm{L}^{-1}$ polyethylene glycol (PEG-4000) and $40.1 \mu \mathrm{M}$ abscisic acid (ABA), respectively. (B,D) Fully mature cotyledonary embryos after eight weeks on solid culture medium and in the TIB, respectively. 


\section{Materials and Methods}

\subsection{Description of the TIB System}

The new bioreactor system consists of a round culture vessel (diameter of $125 \mathrm{~mm}$ and a height of $45 \mathrm{~mm}$ ) and a $500 \mathrm{~mL}$ bottle for holding liquid medium. The covers of the culture vessel and medium bottle are both fitted with $0.22 \mu \mathrm{M}$ sterile polytetrafluoroethylene (PTFE) membrane air filters (Figure 5A). An inlet at the bottom of the vessel offers connectivity to the medium bottle via a silicon tube. Additional silicon tubes link the air filters on top of each medium bottle to a central pipe, which is connected to a pump controlled by an electronic timer. When the pump is switched on, compressed air is blown into the medium bottles and drives the medium upwards into the culture vessels thereby immersing the explants for a pre-set duration. Conversely, gravity-induced flow forces the medium to flow back into the medium bottles once the pump is switched off. Flow back of the medium is aided by the overall arrangement of the system whereby the culture vessels are placed on shelves situated one level above the medium bottles (Figure 5B). Before setting up the experiments, the TIB components along with pre-cut filter paper sheets are placed inside separate heat-sealing sterilization pouches and autoclaved once using a dry cycle of $121^{\circ} \mathrm{C}$ for $15 \mathrm{~min}$. After autoclaving, the TIB components are assembled under a laminar flow hood. The explants are transferred onto a sterile filter paper placed on top of the mesh at the bottom of the culture vessel. During the TIB experiments, the explants were immersed in liquid medium for $1 \mathrm{~min} \cdot \mathrm{h}^{-1}$ throughout the growth period. We used an immersion cycle of one minute per hour because initial testing of the pump indicated that one minute is the actual pump run time required to drive up the medium and fully immerse the explants.
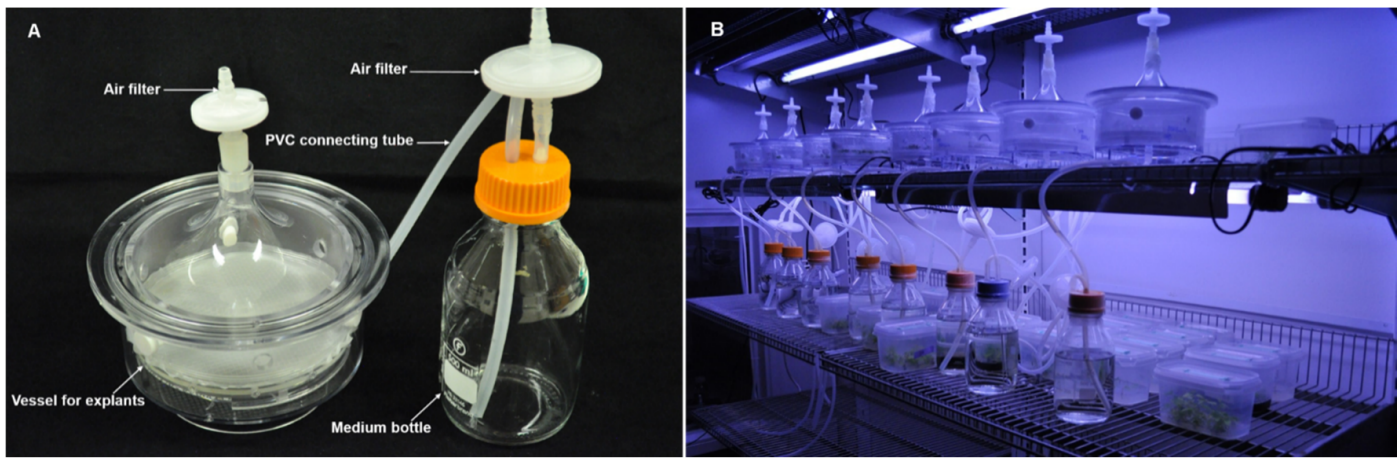

Figure 5. (A) Pictorial representation of the TIB and its components. (B) Arrangement of the TIBs during the course of the experiments.

\subsection{Plant Material and Growth Conditions}

One embryogenic cell line of A. nordmanniana (N.12.9/7.203) was obtained from the company Institut für Pflanzenkultur e.K. (IFP), Solkau, Germany. Additionally, in vitro plants of B. pendula and B. pubescens were obtained from IFP, where they are sold as part of the silvaSELECT clone mixture. The clones were established by the Northwest German Forest Research Station (Nordwestdeutsche Forstliche Versuchsanstalt: NW-FVA, Göttingen, Germany), and are licensed to be sold in Germany under FovG (Forst-Vermehrungsgut-Gesetz). We also obtained two-week-old shoot cultures of Eucalyptus from SweTree Technologies AB, Umeå, Sweden. For proliferation, the embryogenic cultures of cell line N.12.9/7.203 were maintained on half-strength Brown and Lawrence (BLG) medium [8] supplemented with $10 \mathrm{~g} \cdot \mathrm{L}^{-1}$ sucrose and $1.2 \mathrm{mg} \cdot \mathrm{L}^{-1}$ 6-benzylaminopurine (BAP), and grown at $24^{\circ} \mathrm{C}$ under dark conditions for two weeks. After proliferation, the cultures were transferred to BLG medium without hormones and grown at $24{ }^{\circ} \mathrm{C}$ under dark conditions for one week. For somatic embryo maturation, the cultures were transferred to half-strength BLG medium supplemented with $45 \mathrm{~g} \cdot \mathrm{L}^{-1}$ maltose, $50 \mathrm{~g} \cdot \mathrm{L}^{-1}$ polyethylene glycol (PEG-4000), $10.6 \mathrm{mg} \cdot \mathrm{L}^{-1}$ abscisic acid (ABA), and grown at $24^{\circ} \mathrm{C}$ 
for eight weeks under dark conditions. For solid medium culture, the BLG medium was solidified using $3.5 \mathrm{~g} \cdot \mathrm{L}^{-1}$ of Phytagel (Sigma-Aldrich, St. Louis, MO, USA). All the media was sterilized by adjusting its $\mathrm{pH}$ to 5.7 and autoclaving once using a wet cycle of $121^{\circ} \mathrm{C}$ for $20 \mathrm{~min}$. The ABA was prepared as filter-sterilized stock solutions and added after autoclaving and cooling of the medium. During somatic embryo maturation, the medium was refreshed every two weeks.

For Eucalyptus, the shoot cultures were cultivated for two weeks in modified Murashige and Skoog (MS) medium [12] with $550 \mathrm{mg} \cdot \mathrm{L}^{-1} \mathrm{NH}_{4} \mathrm{NO}_{3}$ and $550 \mathrm{mg} \cdot \mathrm{L}^{-1} \mathrm{MgSO}_{4}$ supplemented with $15 \mathrm{~g} \cdot \mathrm{L}^{-1}$ sucrose, $0.15 \mathrm{mg} \cdot \mathrm{mL}^{-1} \mathrm{BAP}$, and $0.01 \mathrm{mg} \cdot \mathrm{mL}^{-1} \mathrm{Naphthaleneacetic} \mathrm{acid} \mathrm{(NAA).} \mathrm{For} \mathrm{solid}$ medium culture, $5 \mathrm{~g} \cdot \mathrm{L}^{-1}$ Phyto agar (Duchefa Biochemie B.V, Harlem, The Netherlands) was added. The explants of B. pendula and B. pubescens were grown on multiplication medium based on McCown's Woody plant medium (WPM). For B. pendula, the WPM medium was supplemented with $20 \mathrm{~g} \cdot \mathrm{L}^{-1}$ sucrose, $1.5 \mathrm{mg} \cdot \mathrm{L}^{-1}$ Zeatin while the medium for $B$. pubescens was supplemented with $2 \mathrm{mg} \cdot \mathrm{L}^{-1}$ BAP, $30 \mathrm{~g} \cdot \mathrm{L}^{-1}$ sucrose $1.5 \mathrm{mg} \cdot \mathrm{L}^{-1}$ Zeatin while the medium for $B$. pubescens was supplemented with $2 \mathrm{mg} \cdot \mathrm{L}^{-1} \mathrm{BAP}, 30 \mathrm{~g} \cdot \mathrm{L}^{-1}$ sucrose. The solid medium for both species was solidified using $6 \mathrm{~g} \cdot \mathrm{L}^{-1}$ Phyto agar [5]. All the media was sterilized by adjusting its $\mathrm{pH}$ to 5.7 and autoclaving a single time using a wet cycle $\left(20 \mathrm{~min}\right.$ at $121^{\circ} \mathrm{C}$ ). During the experiment, the explants of Betula and Eucalyptus were grown under white fluorescent lamps with a $16 \mathrm{~h}$ photoperiod at $25^{\circ} \mathrm{C}$ for 42 days.

\subsection{Experimental Setup and Statistical Analysis}

We compared two culture systems: solid and temporary immersion. Solid medium culture experiments were performed using $92 \times 16 \mathrm{~mm}$ petri dishes (Sarstedt, Nümbrecht, Germany) and ECO2boxes/green filter (Duchefa Biochemie). Each TIB was connected to a bottle containing $400 \mathrm{~mL}$ of liquid medium and the cultures were subjected to $1 \mathrm{~min} \cdot \mathrm{h}^{-1}$ immersions in liquid medium. For B. pendula and B. pubescens, 10 stem explants of $2-3 \mathrm{~cm}$ in length with two shoots were transferred to the TIB and ECO2 boxes, while 20 small shoot clumps were used for Eucalyptus. Approximately 0.9 and $0.78 \mathrm{~g}$ of fresh weight (FW) of embryogenic tissue from cell line N.12.9/7.203 was transferred to the petri dishes and TIB at the start of the experiment, respectively. There were three replicate vessels for each treatment and species. Fresh weight of the explants was recorded at the start (day zero) and end of the culture period for Eucalyptus (2 weeks), A. nordmanniana (11 weeks), B. pendula, and B. pubescens ( 6 weeks). The multiplication rates of Eucalyptus, B. pendula, and B. pubescens were determined by taking the number of shoots at the end of the micropropagation period and dividing by the initial explants. All statistical analyses including calculation of SEM and two-tailed non-paired Student's $t$-tests were performed in Microsoft Excel (Microsoft Corporation, Seattle, WA, USA). The term significant is only used when the change in question is confirmed to be significant $(P<0.05)$ with the $t$-test. Significantly different parameters are indicated using an asterisk $\left({ }^{*}\right)$ on the figures.

\subsection{Acclimatization of in Vitro Plants of B. pendula and B. pubescens to ex Vitro Conditions}

After 42 days of culture, the plants of B. pendula and B. pubescens were induced to form roots using a slightly modified version of the immersion method proposed by [11]. Briefly, we transferred all the plants propagated via solid medium culture into sterile TIBs and left the ones propagated via TIB in their original vessels. Next, all bioreactors were connected to bottles containing $400 \mathrm{~mL}$ of WPM rooting medium supplemented with $0.1 \mathrm{mg} \cdot \mathrm{L}^{-1}$ indole-3-butyric acid (IBA) and $0.05 \mathrm{mg} \cdot \mathrm{L}^{-1}$ Naphthaleneacetic acid (NAA), and without sugar. The elongated shoots were subjected to $1 \mathrm{~min} \cdot \mathrm{h}^{-1}$ immersions in rooting medium under white fluorescent lamps at $25^{\circ} \mathrm{C}$. After $12 \mathrm{~h}$, we transplanted three shoots each of B. pendula and B. pubescens into pots containing a 3:1 soil-to-perlite mixture and sprayed them with WPM medium. The plant trays were placed in a growth chamber under humidity covers and $24 \mathrm{~h}$ light exposure. The shoots were fertilized and watered once and twice a week after the first week, respectively. We did not perform root induction on the shoots of B. pubescens derived from solid multiplication medium because they exhibited actively developing roots after 42 days on this medium. 


\section{Discussion}

In the present study, we evaluated the applicability of a new automated bioreactor operating on the principle of temporary immersion for the micropropagation of B. pendula, B. pubescens, E. grandis $x$ E. urophylla, and A. nordmanniana. We found that propagation of Eucalyptus using the TIB resulted in normal shoot cultures with less callus in the shoots, similar to what has been observed for clones of Eucalyptus derived from the temporary immersion system (TIS) RITA ${ }^{\circledR}$ bioreactor [13]. Compared to solid culture medium, we observed wrinkling of leaves in the plants of $B$. pendula, while those of B. pubescens appeared normal after 42 days of propagation in the TIB system. Manifestations of broad and translucent leaves with a fragile, curly, or wrinkled appearance in plants cultivated under temporary immersion have previously been attributed to hyperdricity [13-16]. Moreover, the study of [15] further reported that plants with symptoms of hyperhydricity seldom survive acclimatization, and those that manage to survive the acclimatization period tend to have an abnormal appearance. However, in our study we found that the plants of $B$. pendula with hyperhydric symptoms acclimated to ex vitro conditions and developed into healthy and morphologically normal plants. Thus, the wrinkled appearance of $B$. pendula leaves might have been the result of a negative response for this particular cultivar towards the immersion frequency of $1 \mathrm{~min} \cdot \mathrm{h}^{-1}$. The use of medium with anti-hyperhydricity agents [17] and the reduction of immersion frequency could perhaps eliminate the hyperhydric symptoms observed in the leaves of B. pendula plants.

The process of micropropagation comprises three sequential steps which start with the establishment of explants followed by the multiplication of explants, rooting, and acclimatization [18]. With the exception of B. pubescens, propagation of B. pendula and Eucalyptus in the TIB resulted in a significant increase in multiplication rate compared to solid medium culture. Overall, the results of the present study are consistent with results from previous studies of pineapple [19], apple root stock [20], and Siraitia grosvenorii [21] indicating that propagation via TIS offers higher rates of shoot multiplication than other systems such as solid and liquid medium culture.

In addition to being used as an experimental system for studying developmental aspects during plant embryogenesis, SE also provides a potential system for the large-scale propagation of plants in automated bioreactors [22]. In our experiments, we found that the maturation frequency of somatic embryos increased significantly when using the TIB for somatic embryogenesis of $A$. nordmanniana. Our SE results are similar to those of [23,24], who found clear differences between cultures of English oak (Quercus robur L.) and Cork oak (Quercus suber L.) cultivated using the RITA $^{\circledR}$ bioreactor and semi-solid culture medium, respectively. The two studies found that, compared to semi-solid culture medium, cultivation of oak cultures using the RITA ${ }^{\circledR}$ system led to increased rates of culture multiplication and embryo maturation. These results were attributed to increased biomass production and the synchronization of somatic embryogenesis in the RITA ${ }^{\circledR}$ system [23]. In conclusion, we report that the bioreactor system could be used for stable micropropagation via shoot multiplication or somatic embryogenesis. These results are encouraging, as part of our current efforts are geared towards optimizing automated somatic embryogenesis for coniferous species-particularly Norway spruce.

Acknowledgments: This work was supported by funding from UPSC Berzelii Centre for Forest Biotechnology and SweTree Technologies (STT). We thank Ioana Gaboreanu and Izabela Dobrowolska (SLU) for technical assistance, Magnus Hertzberg (STT) for critical reading of the manuscript.

Author Contributions: Conceived and designed the experiments: U.E., E.B., A.T. Performed the experiments: E.B. Analyzed the data: E.B., A.T. Contributed reagents/materials: C.S., P.R. Wrote the paper E.B., U.E. All authors have read and approved the final manuscript.

Conflicts of Interest: The authors declare no conflicts of interest. 


\section{References}

1. Etienne, H.; Berthouly, M. Temporary immersion systems in plant micropropagation. Plant Cell Tissue Organ Cult. 2002, 69, 215-231. [CrossRef]

2. Aidun, C.; Egertsdotter, E. Fluidics-based automation of clonal propagation via somatic embryogenesis: Se-fluidics system. In Proceedings of the Iufro Working Party 2.09.02: "Somatic embryogenesis of trees" Conference on "Integrating Vegetative Propagation, Biotechnologies and Genetic Improvement for Tree Production and Sustainable Forest Management", Brno, Czech Republic, 25-28 June 2012; pp. S3-S3.

3. Batish, D.R.; Singh, H.P.; Kohli, R.K.; Kaur, S. Eucalyptus essential oil as a natural pesticide. For. Ecol. Manag. 2008, 256, 2166-2174. [CrossRef]

4. Mokotedi, M.E.; Watt, M.; Pammenter, N. Analysis of differences in field performance of vegetatively and seed-propagated eucalyptus varieties II: Vertical uprooting resistance. South. For. 2010, 72, 31-36.

5. Meier-Dinkel, A. Micropropagation of birches (Betula spp.). In High-Tech and Micropropagation II; Springer: Berlin, Germany, 2012; pp. 40-79.

6. Hynynen, J.; Niemistö, P.; Viherä-Aarnio, A.; Brunner, A.; Hein, S.; Velling, P. Silviculture of birch (Betula pendula Roth and Betula pubescens Ehrh.) in northern europe. Forestry 2010, 83, 103-119. [CrossRef]

7. Lelu-Walter, M.-A.; Thompson, D.; Harvengt, L.; Sanchez, L.; Toribio, M.; Pâques, L.E. Somatic embryogenesis in forestry with a focus on Europe: State-of-the-art, benefits, challenges and future direction. Tree Genet. Genomes 2013, 9, 883-899. [CrossRef]

8. Find, J.; Grace, L.; Krogstrup, P. Effect of anti-auxins on maturation of embryogenic tissue cultures of Nordmanns fir (Abies nordmanniana). Physiol. Plant. 2002, 116, 231-237. [CrossRef] [PubMed]

9. Pedersen, L.B.; Christensen, C.J.; Ingerslev, M. Leaching versus input of nitrogen, potassium and magnesium in different fertilizer regimens in christmas tree stands of Abies nordmanniana in Denmark. Scand. J. For. Res. 2006, 21, 130-142. [CrossRef]

10. Vooková, B.; Kormutak, A. Study of abies somatic embryogenesis and its application. Dendrobiology 2014, 71, 149-157. [CrossRef]

11. Kroin, J. Propagate plants from cuttings using dry-dip rooting powders and water based rooting solutions. Comb. Proc. Intl. Plant Prop. Soc. 2008, 58, 360-372.

12. Murashige, T.; Skoog, F. A revised medium for rapid growth and bio assays with tobacco tissue cultures. Physiol. Plant. 1962, 15, 473-497. [CrossRef]

13. Mc Alister, B.; Finnie, J.; Watt, M.; Blakeway, F. Use of the temporary immersion bioreactor system (RITA $\left.{ }^{\circledR}\right)$ for production of commercial Eucalyptus clones in Mondi Forests (SA). In Liquid Culture Systems for In Vitro Plant Propagation; Springer: Berlin, Germany, 2005; pp. 425-442.

14. Chakrabarty, D.; Dewir, Y.; Hahn, E.; Datta, S.; Paek, K. The dynamics of nutrient utilization and growth of apple root stock ' $\mathrm{M}_{9} \mathrm{EMLA}^{\prime}$ in temporary versus continuous immersion bioreactors. Plant Growth Regul. 2007, 51, 11-19. [CrossRef]

15. Kevers, C.; Franck, T.; Strasser, R.J.; Dommes, J.; Gaspar, T. Hyperhydricity of micropropagated shoots: A typically stress-induced change of physiological state. Plant Cell Tissue Organ Cult. 2004, 77, 181-191. [CrossRef]

16. Welander, M.; Zhu, L.-H.; Li, X.-Y. Factors influencing conventional and semi-automated micropropagation. Propag. Ornam. Plants 2007, 7, 103-111.

17. Marga, F.; Vebret, L.; Morvan, H. Agar fractions could protect apple shoots cultured in liquid media against hyperhydricity. Plant Cell Tissue Organ Cult. 1997, 49, 1-5. [CrossRef]

18. Preece, J.E.; Read, P.E. The Biology of Horticulture: An Introductory Textbook; John Wiley \& Son: Hoboken, NJ, USA, 1993.

19. Escalona, M.; Lorenzo, J.; González, B.; Daquinta, M.; González, J.; Desjardins, Y.; Borroto, C. Pineapple (Ananas comosus L. Merr) micropropagation in temporary immersion systems. Plant Cell Rep. 1999, 18, 743-748. [CrossRef]

20. Zhu, L.-H.; Li, X.-Y.; Welander, M. Optimisation of growing conditions for the apple rootstock M26 grown in RITA containers using temporary immersion principle. In Liquid Culture Systems for In Vitro Plant Propagation; Springer: Berlin, Germany, 2005; pp. 253-261.

21. Yan, H.; Liang, C.; Li, Y. Improved growth and quality of Siraitia grosvenorii plantlets using a temporary immersion system. Plant Cell Tissue Organ Cult. (PCTOC) 2010, 103, 131-135. [CrossRef] 
22. Mamun, N.H.; Egertsdotter, U.; Aidun, C.K. Bioreactor technology for clonal propagation of plants and metabolite production. Front. Biol. 2015, 10, 177-193. [CrossRef]

23. Mallon, R.; Covelo, P.; Vieitez, A.M. Improving secondary embryogenesis in Quercus robur: Application of temporary immersion for mass propagation. Trees 2012, 26, 731-741. [CrossRef]

24. Perez, M.; Bueno, M.A.; Escalona, M.; Toorop, P.; Rodriguez, R.; Canal, M.J. Temporary immersion systems $\left(\right.$ RITA $\left.^{\circledR}\right)$ for the improvement of cork oak somatic embryogeneic culture proliferation and somatic embryo production. Trees 2013, 27, 1277. [CrossRef]

(c) 2017 by the authors. Licensee MDPI, Basel, Switzerland. This article is an open access article distributed under the terms and conditions of the Creative Commons Attribution (CC BY) license (http:/ / creativecommons.org/licenses/by/4.0/). 